Trauma Berufskrankh 2008 · 10 [Suppl 2]:219-225

DOI 10.1007/s10039-007-1321-5

Online publiziert: 26. April 2008

(c) Springer Medizin Verlag 2008

\author{
S. Hungerer $\cdot$ P. Augat $\cdot V$. Bühren \\ BG-Unfallklinik, Murnau
}

\section{Elektromagnetische Verfahren bei Knochenheilungsstörungen} Evidenz dieser additiven Maßnahme
Der Zusammenhang von elektrischen Potenzialen zur Unterstützung der Knochenheilung wurde erstmalig im 19. Jahrhundert beschrieben [34, 44]. Dieser Effekt ist jedoch erst wiederentdeckt worden mit der Entdeckung der piezoelektrischen Eigenschaften von Knochen sowie Kollagen $[66,75]$ und den bioelektrischen Eigenschaften von vitalem Knochen [5]. Das Wolff-Gesetz des deutschen Anatoms und Chirurgen Julius Wolff (1835-1902) beschreibt die Eigenschaft von Knochen, sich unter Druckbelastung auf- und unter Zugbelastung abzubauen. Die Übertragung von mechanischen Reizen in elektrische Impulse auf zellulärer Ebene wird als elektromechanische Kopplung bezeichnet und ist der Schlüssel zur Umbaufähigkeit des Knochens z. B. nach Verletzungen [7, 67]. An diesem Punkt setzten die verschiedenen Techniken der Elektrostimulation zur Unterstützung der Knochenheilung an.

In der klinischen Anwendung finden sich 3 verschiedene Techniken der Elektrostimulation:

Direkte Stromapplikation mit implantierbaren Elektroden und meist subkutan implantierbarer Stromquelle. Sie wird auch als DC-Verfahren (DC: „direct current") bezeichnet. Sie findet v. a. bei Spondylodeseoperationen der Wirbelsäule Anwendung. Eine Knochenneubildung ist bei Gleichstromgeräten lediglich an der Kathode (Pluspol) zu beobachten.
Perkutane Elektrostimulation mittels Klebeelektroden und externem Steuerungsgerät. Sie wird auch als CCVerfahren (CC: „capacitive coupling“) bezeichnet. Die perkutane Stromzuleitung hat den Nachteil der begrenzten Eindringtiefe und möglichen lokalen Reaktion.

Induktive Verfahren (IC-Verfahren, IC: „inductive coupling "). Hier haben sich v. a. die pulsierenden elektromagnetischen Felder (PEMF) etabliert. Bei ihnen werden Magnetfelder über externe Magnetfeldspulen appliziert, die in Abhängigkeit von Frequenz und Amplitude des Signals elektromagnetische Felder induzieren.

Die zuletzt genannten elektromagnetischen Verfahren werden im Folgenden genauer betrachtet.

\section{Experimentelle Daten}

Mittels nichtthermischen niederfrequenten Magnetfeldern über das Transformatorenprinzip wurden erstmals 1968 Struktur bildende Wechselfelder am Knochen induziert und in der Folge systematisch untersucht. Eine Vielzahl älterer experimenteller Untersuchungen wurde von Bassett [6] zusammengefasst und eine andere Übersichtsarbeit der Wirkmechanismen von Aaron et al. [1] veröffentlicht. Es wurden In-vitro-Auswirkungen auf die Proteoglykansynthese beschrieben, den Kalziumstoffwechsel, die Kollagensynthese und die zellulären Signalwege. In neueren Arbeiten wurden diese Mechanismen im Hinblick auf die Zelldifferenzie- rung von Osteoblasten weiter untersucht und bestätigt $[64,74,76]$. Der Einfluss von PEMF auf die Genexpression von $c$-fos und $c-m y c$ in Osteoblasten konnte nachgewiesen werden [18], ebenso die Eigenschaft von PEMF, die Zellaktivität von Osteoblasten zu erhöhen und die Synthese von extrazellulärer Matrix zu stimulieren [49]. Ähnliche Effekte wurden für Chondrozyten beschrieben [16]. Bei Osteoklasten konnten dagegen eine Beschleunigung der Apoptoserate nachgewiesen werden [15] sowie die Modulation von Zytokinen in der Entwicklung von Osteoklasten [14]. In einer Untersuchung von Li et al. [47] wurde der Effekt von Ultraschall und PEMF verglichen, und es wurde gezeigt, dass beide Verfahren den intrazellulären Kalziumgehalt erhöhen und den intrazellulären Botenstoff Calmodulin aktivieren. An einem In-vitro-Modell mit Zellen aus humanen Pseudarthrosen konnte eine Stimulation der parakrinen Faktoren für Knochenwachstum mit PEMF-Behandlung aufgezeigt werden [32].

In älteren tierexperimentellen Studien wurden die Grundlage gebildet und der Effekt von PEMF nachgewiesen [10, 29, $33,42]$. In neueren In-vivo-Studien konnte die Wirkung von PEMF auf eine Beschleunigung der Frakturheilung nachgewiesen werden, so z. B. bei Fibulaosteotomie der Ratte $[38,56]$ oder am Modell der Kallusdistraktion beim Hasen $[25,26]$ und Kaninchen [39]. 


\begin{tabular}{|c|c|c|c|c|c|c|c|}
\hline Zitat & Jahr & Indikation & $\begin{array}{l}\text { Zusätzliche } \\
\text { Therapie }\end{array}$ & PEMF-Parameter & $\begin{array}{l}\text { Behandlungs- } \\
\text { parameter }\end{array}$ & Therapie-/Kontrollgruppe (n) & $\begin{array}{l}\text { EBM- } \\
\text { Level }\end{array}$ \\
\hline $\begin{array}{l}\text { Borsalino } \\
\text { et al. [11] }\end{array}$ & 1988 & $\begin{array}{l}\text { Intertrochantäre } \\
\text { Osteotomie }\end{array}$ & Keine & $\begin{array}{l}75 \mathrm{~Hz} \\
2,5 \mathrm{mV} \\
1,8 \mathrm{mT} \\
\text { (Fa. IGEA) }\end{array}$ & $\begin{array}{l}8 \mathrm{~h} / \text { Tag } \\
90 \text { Tage }\end{array}$ & $\begin{array}{l}\text { 16/16 } \\
0,8 \text { vs. } 0,31 \text { Punkte Kallus Tag } 40 \\
1,9 \text { vs. 1,4 Punkte Kallus Tag } 90\end{array}$ & I \\
\hline $\begin{array}{l}\text { Capanna et } \\
\text { al. [13] }\end{array}$ & 1994 & $\begin{array}{l}\text { Autologe Kno- } \\
\text { chentransplan- } \\
\text { tation }\end{array}$ & $\begin{array}{l}\text { Osteosynthese } \\
\text { Platten, } \\
\text { im Nagel }\end{array}$ & $\begin{array}{l}75 \mathrm{~Hz} \\
3,0 \mathrm{mV} \\
\text { (Fa. IGEA) }\end{array}$ & $\begin{array}{l}4 \mathrm{~h} / \mathrm{Tag} \\
-360 \text { Tage }\end{array}$ & $\begin{array}{l}24 / 23 \\
54 \text { vs. } 56 \% \text { Konsolidierung } \\
8,5 \text { vs. } 9,4 \text { Monate }\end{array}$ & I \\
\hline $\begin{array}{l}\text { Eyres } \\
\text { et al. [22] }\end{array}$ & 1996 & Kallusdistraktion & Keine & $\begin{array}{l}15 \mathrm{~Hz} \\
1,6 \mathrm{mT} \\
\text { (Fa. ElectroBiology) }\end{array}$ & $4 \mathrm{~h} / \mathrm{Tag}$ & $\begin{array}{l}7 / 6 \\
13 \text { vs. } 54 \% \text { Abnahme } \\
\text { der Knochendichte }\end{array}$ & (I) IV \\
\hline $\begin{array}{l}\text { Fontanesi } \\
\text { et al. [24] }\end{array}$ & 1986 & $\begin{array}{l}\text { Frakturheilung } \\
\text { Tibia }\end{array}$ & Gips & $\begin{array}{l}75 \mathrm{~Hz} \\
2,5 \mathrm{mV} \\
2 \mathrm{mT} \\
\text { (Fa. IGEA, Howmedica) }\end{array}$ & $\begin{array}{l}\text { 8-10 h/Tag } \\
70-210 \text { Tage }\end{array}$ & 20/20 Konsolidierung & III \\
\hline $\begin{array}{l}\text { Gonzales et } \\
\text { al. [30] }\end{array}$ & 2005 & Kallusdistraktion & Keine & $\begin{array}{l}75 \mathrm{~Hz} \\
3,5 \mathrm{mV} \\
\text { (Fa. IGEA) }\end{array}$ & $\begin{array}{l}8 \mathrm{~h} / \mathrm{Tag} \\
? ?\end{array}$ & $\begin{array}{l}30 \text { Extremitätenpaare } \\
\text { Therapie }>\text { Kontrolle } \\
\text { Knochendichte Kallus }\end{array}$ & III \\
\hline $\begin{array}{l}\text { Mammi et } \\
\text { al. [51] }\end{array}$ & 1993 & Tibiaosteotomie & Keine & $\begin{array}{l}75 \mathrm{~Hz} \\
3 \mathrm{mV} \\
\text { (Fa. IGEA) }\end{array}$ & $\begin{array}{l}8 \mathrm{~h} / \mathrm{Tag} \\
60 \text { Tage }\end{array}$ & $\begin{array}{l}20 / 20 \\
\text { Therapie > Kontrolle } \\
\text { Konsolidierung }\end{array}$ & I \\
\hline $\begin{array}{l}\text { Dhawan et } \\
\text { al. [19] }\end{array}$ & 2004 & $\begin{array}{l}\text { Arthrodesen } \\
\text { talonavikular (tn), } \\
\text { subtalar (st) und } \\
\text { kalkaneokuboid } \\
\text { (cc) }\end{array}$ & Keine & $\begin{array}{l}\text { ?? } \\
\text { (Fa. ElectroBiology) }\end{array}$ & $\begin{array}{l}12 \mathrm{~h} / \mathrm{Tag} \\
\sim 91 \text { Tage }\end{array}$ & $\begin{array}{l}\text { st } 33+4 / 22+5 \\
12,9 \text { vs. } 14,5 \text { Wochen } \\
\text { Konsolidierung } \\
\text { tn } 19 / 23 \\
12,2 \text { vs. } 17,6 \text { Wochen } \\
\text { Konsolidierung } \\
\text { cc } 21 / 19 \\
13,1 \text { vs. } 17,7 \text { Wochen } \\
\text { Konsolidierung }\end{array}$ & (I) III? \\
\hline $\begin{array}{l}\text { Saltzman } \\
\text { et al. [61] }\end{array}$ & 2004 & $\begin{array}{l}\text { Fuß- und Sprung- } \\
\text { gelenkarthrodesen }\end{array}$ & Keine & $\begin{array}{l}\text { ? } \\
\text { (Fa. ElectroBiology) }\end{array}$ & $\begin{array}{l}? \\
150-810 \text { Tage }\end{array}$ & $\begin{array}{l}\text { 19/keine } \\
\text { 26\% Durchbau }\end{array}$ & IV \\
\hline
\end{tabular}

\section{Klinische Studien}

Die PEMF sind seit 1979 von der amerikanischen Food and Drug Administration (FDA) als additives Verfahren zur Unterstützung der Knochenheilung bei Pseudarthrosen und Arthrodesen zugelassen. Seit 1970 kommen in Deutschland elektromagnetische Verfahren in der Therapie von Pseudarthrosen und verzögerten Knochenheilung zur klinischen Anwendung $[43,45]$. Seither wurden eine Vielzahl von klinischen Studien publiziert, die eine Therapie mit PEMF propagieren, jedoch sind diese, gemessen an den heutigen Standards der evidenzbasierten Medizin, z. T. von sehr unterschiedlicher Qualität (http://www.cebm.net). Im Folgenden werden die klinischen Studien nach ihren Anwendungsgebieten der PEMF-Therapie zur Unterstützung der Knochenheilung, Behandlung von Pseu- darthrosen oder Verbesserung operativer Ergebnisse nach Knocheneingriffen aufgeteilt. Es werden nur Publikationen berücksichtigt, die in „peer reviewed“-Zeitschriften veröffentlicht wurden, und nur die Sprachen Englisch, Deutsch und Französisch. Veröffentlichungen im Rahmen von Kongressen, Buchveröffentlichungen usw. wurden nicht mit einbezogen.

Die knöcherne Heilung nach Frakturen und Osteotomien stellt nur in einem geringen Prozentsatz der Patienten ein Problem dar. Die große Zahl der Patienten macht hieraus aber einen relevanten Faktor. Es gibt 2 ältere Studien mit zufrieden stellenden Designs von Borsalino et al. [11] und Mammi et al. [51], in denen die Frakturheilung nach Osteotomien an der unteren Extremität untersucht wurde (• Tab. 1). Die PEMF-Therapie wurde auch zur Unterstützung der knöchernen Heilung nach Versteifungsoperati- on angewendet. Die Arbeit von Dhawan et al. [19] wurde zwar als Level I eingestuft [41], es wurden jedoch nicht die Patienten $(n=64)$, sondern die einzelnen Gelenke $(n=144)$ als Fall gezählt und somit bei einem Patienten mehrere Gelenke in die Auswertung einbezogen. Damit können mehrere Ergebnisse abhängig von einem Patienten sein, und das Studiendesign entspricht somit nicht den Anforderungen einer Level-I-Studie.

In der Behandlung von verzögerten Knochenbrüchen und Pseudarthrosen gibt es eine Vielzahl von klinischen Studien (- Tab. 2). Als Level I werden 2 davon gehandelt. Vom Studiendesign und der Anzahl der Patienten ist die Studie von Sharrard [68] mit Vorbehalt als LevelI-Studie zu werten, da nicht klar hervorgeht, wie die Anzahl der Gruppen errechnet wurde und ob die Patienten randomisiert wurden. Der Studie von Barker et al. 
[4] ermangelt es ebenfalls an dem klaren Studiendesign, und sie muss daher - bei einer Gesamtzahl von 9 behandelten $\mathrm{Pa}$ tienten - eher als Level-IV-Studie angesehen werden.

Ein weiteres Feld der PEMF-Verfahren ist die Unterstützung der knöchernen Konsolidierung nach Fusionsoperationen an der Wirbelsäule (• Tab. 3). 2 Übersichtsarbeiten beschäftigten sich jeweils mit dieser Applikation [3, 28] und versuchten aus der gesamten Bandbreite der elektrischen Stimulationsmöglichkeiten Schlüsse über die Effizienz dieser additiven Therapieformen zu ziehen. Es finden sich jedoch 2 Studien zur PEMFTherapie nach Spondylodeseoperationen mit Studiendesign, die dem EBM-Level I entsprechen und von den Patientenzahlen ausreichend erscheinen. In der Arbeit von Linovitz et al. [48] wurde eine Erfolgsrate der knöchernen Konsolidierung von $64 \%$ vs. $43 \%$ berichtet, Mooney [57] beschrieb eine Erfolgsrate von $92 \%$ vs. $65 \%$ gegenüber der Kontrollgruppe.

Angrenzende Gebiete zur Unterstützung der Knochenneubildung sind die aseptische Hüftkopfnekrose oder aseptische Prothesenlockerung. Massari et al. [54] untersuchten die Wirkung von PEMF auf die Hüftkopfnekrose, und in dieser Level-IV-Studie wurde ein positiver Effekt von PEMF in den Stadien Ficat I und II beschrieben. In einer Level-IV-Studie konnten Kennedy et al. [40] mit PEMFTherapie eine Revision einer gelockerten Hüftprothese in $53 \%$ der Fälle $(n=19)$ hinauszögern im Vergleich zur Placebokontrolle mit $11 \%$ der Fälle $(n=18)$.

\section{Diskussion}

Mit 3 Veröffentlichungen wurde in den letzten Jahren versucht, die elektrische und elektromagnetische Stimulation in Form von Metaanalysen zusammenzufassen, um eine Aussage über die Wirksamkeit treffen zu können [2, 3, 31]. Damit wurden die eingangs erwähnten Techniken der Stimulation (DC, CC, IC) als gleichwertig angesehen. Eine Zusammenfassung in dieser Art ist sicherlich kritisch zu sehen, obwohl diese Arbeiten eine gute Übersicht über die klinischen Studien geben. Abgesehen von der Vergleichbarkeit der oben genannten Verfahren (DC,CC,

Trauma Berufskrankh 2008 - 10[Suppl 2]:219-225 DOI 10.1007/s10039-007-1321-5

(C) Springer Medizin Verlag 2008

\section{S. Hungerer $\cdot$ P. Augat $\cdot V$. Bühren \\ Elektromagnetische Verfahren bei Knochenheilungsstörungen. Evidenz dieser additiven Maßnahme}

\section{Zusammenfassung}

Elektromagnetisch induzierte Wechselfelder („pulsed electromagnetic fields", PEMF) finden als adjuvantes Verfahren zur Stimulation der Knochenheilung bereits seit Jahrzehnten Anwendung. In einer Vielzahl von experimentellen Studien konnten eine Reihe möglicher Wirkmechanismen aufgezeigt werden. Im klinischen Alltag fanden PEMF Anwendung in der Unterstützung der Knochenbruchheilung, bei Osteotomien, Kallusdistraktionen, Spondylodesenoperationen, Arthrodesen oder der Therapie von verzögerter Knochenbruchheilung bzw. Pseudarthrosen langer Röhrenknochen. Die Vorteile der PEMFTherapie bestehen im Fehlen von Nebenwirkungen sowie der Möglichkeit, diese nichtinvasive Technik als zusätzliches Verfahren ein-

\section{Electromagnetic procedure for impaired bone healing. Evidence relating to this additive method}

\section{Abstract}

Pulsed electromagnetic fields (PEMFs) have been used for decades to stimulate bone healing. Numerous experimental studies have highlighted a variety of possible mechanisms of effect. In daily clinical routine, PEMFs have been used to accelerate bone healing after fractures, after osteotomies, for callus distraction, and after arthrodesis and spondylodesis operations. PEMFs have also been used as an alternative to revision surgery in the treatment of delayed union and pseudarthrosis of long bones. The advantages of PEMFs are the almost complete lack of any known adverse effects and the option of applying this noninvasive therapy to promote zusetzen, um bei Problempatienten die Heilungschancen zu erhöhen. Wie alle klinischen Therapieverfahren müssen sich auch die elektromagnetischen Verfahren an den Kriterien evidenzbasierter Medizin messen lassen. Im folgenden Artikel werden die „peer reviewed"-Publikationen zur Knochenheilung und PEMF-Therapie der letzten Jahre aufgelistet und entsprechend den Kriterien der evidenzbasierten Medizin eingestuft und bewertet.

\section{Schlüsselwörter}

Stimulation der Knochenheilung .

Elektromagnetische Stimulation .

PEMF („pulsed electromagnetic fields") .

Elektrostimulation · Evidenzbasierte Medizin the osseous consolidation in problem cases. Nevertheless, these therapeutic electromagnetic field procedures need to prove their efficacy when measured against evidencebased criteria. In the present paper, the peerreviewed articles on bone healing and the efficacy of PEMFs are summarized and then classified and evaluated in terms of level of evidence.

\section{Keywords \\ Stimulation of bone healing .}

Electromagnetic stimulation - PEMF (pulsed electromagnetic fields) $\cdot$ Electrical stimulation · Evidence-based medicine 


\begin{tabular}{|c|c|c|c|c|c|c|c|}
\hline Zitat & Jahr & Indikation & $\begin{array}{l}\text { Zusätzliche } \\
\text { Therapie }\end{array}$ & PEMF-Parameter & $\begin{array}{l}\text { Behandlungs- } \\
\text { parameter }\end{array}$ & $\begin{array}{l}\text { Therapie-/Kontrollgruppe (n) } \\
\text { Ergebnis Therapie } \\
\text { vs. Kontrolle [\%] }\end{array}$ & $\begin{array}{l}\text { EBM- } \\
\text { Level }\end{array}$ \\
\hline $\begin{array}{l}\text { Barker } \\
\text { et al. [4] }\end{array}$ & 1984 & Pseudarthrosen & Gips & $\begin{array}{l}15 \mathrm{~Hz} \\
1,5 \mathrm{mT} \\
(\mathrm{NN})\end{array}$ & $\begin{array}{l}\text { 12-16 h } \\
\text { (Minimum } 1 \mathrm{~h} \text { ) } \\
\text { 168 Tage }\end{array}$ & $\begin{array}{l}9 / 7 \\
55 \% \text { vs. } 71 \% \text { Konsolidierung }\end{array}$ & (I)IV \\
\hline $\begin{array}{l}\text { Bassett } \\
\text { et al. [8] }\end{array}$ & 1981 & Pseudarthrosen & Gips & $\begin{array}{l}15 \mathrm{~Hz} \\
0,2 \mathrm{mT} \\
\text { Fa. ElectroBiology) }\end{array}$ & $\begin{array}{l}10 \mathrm{~h} / \text { Tag } \\
\sim 150 \text { Tage }\end{array}$ & $\begin{array}{l}\text { 125/keine } \\
87 \% \text { Konsolidierung }\end{array}$ & IV \\
\hline $\begin{array}{l}\text { Bassett } \\
\text { et al. [9] }\end{array}$ & 1982 & Pseudarthrosen & Spongiosa & $\begin{array}{l}15 \mathrm{~Hz} \\
0,2 \mathrm{mT} \\
\text { Fa. ElectroBiology) }\end{array}$ & $\begin{array}{l}10 \mathrm{~h} / \text { Tag } \\
\sim 120 \text { Tage }\end{array}$ & $\begin{array}{l}\text { 83/keine } \\
\text { 87\% Konsolidierung }\end{array}$ & IV \\
\hline $\begin{array}{l}\text { De Haas } \\
\text { et al. [17] }\end{array}$ & 1980 & Pseudarthrose & Keine & $\begin{array}{l}1 \mathrm{~Hz} \\
15-30 \mathrm{mT}\end{array}$ & $\begin{array}{l}20 \mathrm{~h} / \text { Tag } \\
28-56 \text { Tage }\end{array}$ & $\begin{array}{l}17 / 23 \\
83 \text { vs. } 88 \% \text { Konsolidierung }\end{array}$ & III \\
\hline $\begin{array}{l}\text { Dunn u. } \\
\text { Rush [21] }\end{array}$ & 1984 & $\begin{array}{l}\text { Pseudarthrosen- } \\
\text { osteotomie }\end{array}$ & Spongiosa & $\begin{array}{l}? \\
(\mathrm{NN})\end{array}$ & $\begin{array}{l}10 \mathrm{~h} / \mathrm{Tag} \\
60-360 \text { Tage }\end{array}$ & $\begin{array}{l}\text { 35/keine } \\
81 \% \text { Konsolidierung }\end{array}$ & IV \\
\hline $\begin{array}{l}\text { Fontanesi } \\
\text { et al. [23] }\end{array}$ & 1983 & $\begin{array}{l}\text { Delayed union } \\
\text { Pseudarthrosen }\end{array}$ & $\begin{array}{l}\text { Gips } \\
\text { Fixateur externe } \\
\text { Platte }\end{array}$ & $\begin{array}{l}75 \mathrm{~Hz} \\
\text { (Fa. Biostim) }\end{array}$ & $\begin{array}{l}12 \mathrm{~h} / \text { Tag } \\
60-180 \text { Tage }\end{array}$ & $\begin{array}{l}35 / \text { keine } \\
88,5 \% \text { Konsolidierung }\end{array}$ & IV \\
\hline $\begin{array}{l}\text { Freedman } \\
\text { [27] }\end{array}$ & 1985 & Delayed union & Gips & $\begin{array}{l}75 \mathrm{~Hz} \\
\text { (Fa. ElectroBiology) }\end{array}$ & $\begin{array}{l}12 \mathrm{~h} / \mathrm{Tag} \\
60-300 \text { Tage }\end{array}$ & $\begin{array}{l}\text { 12/keine } \\
17 \% \text { Konsolidierung }(n=2)\end{array}$ & IV \\
\hline $\begin{array}{l}\text { Heckman } \\
\text { et al. [35] }\end{array}$ & 1981 & Pseudarthrose & $\begin{array}{l}\text { Spongiosa } \\
\text { Gips }\end{array}$ & $\begin{array}{l}15 \mathrm{~Hz} \\
\text { (Fa. ElectroBiology) }\end{array}$ & $\begin{array}{l}12 \mathrm{~h} / \text { Tag } \\
90-120 \text { Tage }\end{array}$ & $\begin{array}{l}\text { 149/keine } \\
64 \% \text { Konsolidierung }\end{array}$ & IV \\
\hline $\begin{array}{l}\text { Hinsenkamp } \\
\text { et al. [36] }\end{array}$ & 1985 & Pseudarthrosen & Keine & $\begin{array}{l}15 \mathrm{~Hz} \\
\text { (Fa. ElectroBiology) }\end{array}$ & $\begin{array}{l}12 \mathrm{~h} / \mathrm{Tag} \\
?\end{array}$ & $\begin{array}{l}267(308) / \text { keine } \\
72 \% \text { Konsolidierung }\end{array}$ & IV \\
\hline Holmes [37] & 1994 & $\begin{array}{l}\text { Pseudarthrose } \\
\text { Delayed union }\end{array}$ & Gips & $\begin{array}{l}15 \mathrm{~Hz} \\
0-2 \mathrm{mT} \\
\text { (NN) }\end{array}$ & $8-10 \mathrm{~h} / \mathrm{Tag}$ & $\begin{array}{l}\text { 9/keine } \\
\text { 100\% Konsolidierung }\end{array}$ & IV \\
\hline Lechner [46] & 1981 & Pseudarthrose & $\begin{array}{l}\text { Spongiosa } \\
\text { Invasiver Überträger }\end{array}$ & $\begin{array}{l}22-30 \mathrm{~Hz} \\
3-5 \mathrm{mT}\end{array}$ & $\begin{array}{l}3 \mathrm{~h} / \text { Tag } \\
42-? ? \text { Tage }\end{array}$ & $\begin{array}{l}\text { 319/keine } \\
\text { 93,6\% Konsolidierung }\end{array}$ & IV \\
\hline $\begin{array}{l}\text { Lynch u. Mac } \\
\text { Auley [50] }\end{array}$ & 1985 & Pseudarthrosen & Gips & $1,0-1,5 \mathrm{mV}$ & $\begin{array}{l}10 \mathrm{~h} / \mathrm{Tag} \\
56-252 \text { Tage }\end{array}$ & $\begin{array}{l}\text { 26/keine } \\
88,5 \% \text { Konsolidierung }\end{array}$ & IV \\
\hline $\begin{array}{l}\text { Marcer et al. } \\
\text { [52] }\end{array}$ & 1984 & Pseudarthrosen & Fixateur externe & $\begin{array}{l}15 \mathrm{~Hz} \\
15 \mathrm{mV} \\
\text { (Fa. ElectroBiology) }\end{array}$ & $10 \mathrm{~h} / \mathrm{Tag}$ & $\begin{array}{l}\text { 147/keine } \\
\text { 73\% Konsolidierung }\end{array}$ & IV \\
\hline $\begin{array}{l}\text { O'Connor } \\
{[58]}\end{array}$ & 1985 & Pseudarthrosen & $\begin{array}{l}\text { Gips } \\
5 \text { Patienten } \\
\text { mit Operation }\end{array}$ & $\begin{array}{l}15 \mathrm{~Hz} \\
1,0-1,5 \mathrm{mV} \\
\text { (Fa. ElectroBiology) }\end{array}$ & $12 \mathrm{~h} / \mathrm{Tag}$ & $\begin{array}{l}\text { 30/keine } \\
88 \% \text { Konsolidierung }\end{array}$ & IV \\
\hline $\begin{array}{l}\text { Schmidt- } \\
\text { Neuerburg } \\
{[63]}\end{array}$ & 2001 & Pseudarthrosen & Spongiosaplastik & $\begin{array}{l}20 \mathrm{~Hz} \\
700 \mathrm{mV} \\
3-5 \mathrm{mT} \\
\text { (Fa. GEOT) }\end{array}$ & $\begin{array}{l}\text { 2,25-3 h/Tag } \\
\text { 180 Tage }\end{array}$ & $\begin{array}{l}\text { 61/keine } \\
95 \% \text { Konsolidierung }\end{array}$ & IV \\
\hline $\begin{array}{l}\text { Sedel et al. } \\
\text { [65] }\end{array}$ & 1982 & $\begin{array}{l}\text { Pseudarthrosen, } \\
\text { z. T. kongenital }\end{array}$ & $?$ & $\begin{array}{l}? \\
\text { (Fa. ElectroBiology) }\end{array}$ & 30-180 Tage & $\begin{array}{l}\text { 37/keine } \\
84 \% \text { Konsolidierung }\end{array}$ & IV \\
\hline $\begin{array}{l}\text { Sharrard } \\
\text { et al. [69] }\end{array}$ & 1982 & Pseudarthrosen & Gips & $\begin{array}{l}15 \mathrm{~Hz} \\
1,5 \mathrm{mV} \\
\text { (NN) }\end{array}$ & $\begin{array}{l}12-16 \mathrm{~h} / \text { Tag } \\
90-? ? \text { Tgae }\end{array}$ & $\begin{array}{l}\text { 53/keine } \\
72 \% \text { Konsolidierung }\end{array}$ & IV \\
\hline $\begin{array}{l}\text { Sharrard } \\
{[68]}\end{array}$ & 1990 & Pseudarthrosen & Gips & $\begin{array}{l}15 \mathrm{~Hz} \\
(\mathrm{NN})\end{array}$ & $\begin{array}{l}12 \mathrm{~h} / \mathrm{Tag} \\
\sim 84 \text { Tage }\end{array}$ & $\begin{array}{l}20 / 25 \\
40 \text { vs. } 14 \% \text { Konsolidierung }\end{array}$ & (I) \\
\hline $\begin{array}{l}\text { Simonis } \\
\text { et al. [72] }\end{array}$ & 1984 & Pseudarthrosen & Fixateur externe & $\begin{array}{l}23 \mathrm{~Hz} \\
1,4 \mathrm{mT} \\
\text { (St. Thomas Hosp.) }\end{array}$ & $\begin{array}{l}12-16 \mathrm{~h} / \text { Tag } \\
90-240 \text { Tage }\end{array}$ & $\begin{array}{l}\text { 15/keine } \\
87 \% \text { Konsolidierung }\end{array}$ & IV \\
\hline $\begin{array}{l}\text { Simonis } \\
\text { et al. [73] }\end{array}$ & 2003 & Pseudarthrosen & Fixateur externe & $\begin{array}{l}23 \mathrm{~Hz} \\
1,4 \mathrm{mT} \\
(\mathrm{NN})\end{array}$ & $\begin{array}{l}>14 \mathrm{~h} / \mathrm{Tag} \\
180 \text { Tage }\end{array}$ & $\begin{array}{l}18 / 16 \\
89 \text { vs. } 50 \% \text { Konsolidierung }\end{array}$ & 1 \\
\hline
\end{tabular}




\begin{tabular}{|c|c|c|c|c|c|c|c|}
\hline Zitat & Jahr & Indikation & $\begin{array}{l}\text { Zusätzliche } \\
\text { Therapie }\end{array}$ & PEMF-Parameter & $\begin{array}{l}\text { Behandlungs- } \\
\text { parameter }\end{array}$ & $\begin{array}{l}\text { Therapie-/Kontroll- } \\
\text { gruppe (n) }\end{array}$ & EBM-Level \\
\hline Bose [12] & 2001 & $\begin{array}{l}\text { Spondylodese lumbal } \\
\text { PLIF }\end{array}$ & Korsett & $\begin{array}{l}? \\
\text { (Fa. Orthofix) }\end{array}$ & $\begin{array}{l}4 \mathrm{~h} / \mathrm{Tag} \\
?\end{array}$ & $\begin{array}{l}\text { 48/keine } \\
98 \% \text { Fusion }\end{array}$ & IV \\
\hline $\begin{array}{l}\text { Di Silvestre } \\
\text { u. Savini [20] }\end{array}$ & 1992 & $\begin{array}{l}\text { Spondylodese lumbo- } \\
\text { sakral }\end{array}$ & Gipskorsett & $\begin{array}{l}75 \mathrm{~Hz} \\
3,5 \mathrm{mV} \\
\text { (Fa. IGEA) }\end{array}$ & $\begin{array}{l}10-12 \mathrm{~h} / \mathrm{Tag} \\
55 \text { Tage }\end{array}$ & $\begin{array}{l}\text { 31/keine } \\
96 \% \text { Fusion }\end{array}$ & IV \\
\hline $\begin{array}{l}\text { Linovitz } \\
\text { et al. [48] }\end{array}$ & 2002 & $\begin{array}{l}\text { Spondylodese lumbal } \\
\text { PLIF }\end{array}$ & Keine & $\begin{array}{l}77 \mathrm{~Hz} \\
0,02-0,04 \mathrm{mT} \\
\text { (Fa. Orthologic) }\end{array}$ & $\begin{array}{l}0,5 \mathrm{~h} / \mathrm{Tag} \\
270 \text { Tage }\end{array}$ & $\begin{array}{l}104 / 97 \\
64 \text { vs. } 43 \% \text { Fusion }\end{array}$ & 1 \\
\hline Marks [53] & 2000 & Spondylodese lumbal & Korsett & $\begin{array}{l}? \\
\text { (Fa. Orthofix) }\end{array}$ & $\begin{array}{l}4 \mathrm{~h} / \mathrm{Tag} \\
?\end{array}$ & $\begin{array}{l}42 / 19 \\
98 \% \text { vs. } 53 \% \text { Fusion }\end{array}$ & (III) IV \\
\hline Mooney [57] & 1990 & Spondylodese lumbal & Korsett & $\begin{array}{l}1,5 \mathrm{~Hz} \\
0,18 \mathrm{mT} \\
(\mathrm{NN})\end{array}$ & $\begin{array}{l}8 \mathrm{~h} / \mathrm{Tag} \\
?\end{array}$ & $\begin{array}{l}98 / 95 \\
92 \text { vs. } 65 \% \text { Fusion }\end{array}$ & 1 \\
\hline $\begin{array}{l}\text { Savini et al. } \\
\text { [62] }\end{array}$ & 1990 & Spondylodese lumbal & Gipskorsett & $\begin{array}{l}75 \mathrm{~Hz} \\
2,5 \mathrm{mV} \\
\text { (Fa. IGEA) }\end{array}$ & $\begin{array}{l}10 \mathrm{~h} / \mathrm{Tag} \\
60 \text { Tage }\end{array}$ & $\begin{array}{l}\text { 15/keine } \\
\text { 93\% Fusion }\end{array}$ & IV \\
\hline $\begin{array}{l}\text { Simmons } \\
\text { et al. [71] }\end{array}$ & 2004 & $\begin{array}{l}\text { Pseudarthrose nach } \\
\text { lumbaler Fusion }\end{array}$ & Keine & $\begin{array}{l}16-585 \mu \mathrm{T} \\
\text { (Fa. Orthofix) }\end{array}$ & $\begin{array}{l}2 \mathrm{~h} / \text { Tag } \\
90 \text { Tage }\end{array}$ & $\begin{array}{l}\text { 100/keine } \\
67 \% \text { Fusion }\end{array}$ & IV \\
\hline Simmons [70] & 1985 & $\begin{array}{l}\text { Spondylodese lumbal } \\
\text { PLIF }\end{array}$ & Keine & $\begin{array}{l}2 \mathrm{~Hz} \\
0,4 \mathrm{mT} \\
\text { (Fa. ElectroBiology) }\end{array}$ & $8-10 \mathrm{~h} / \mathrm{Tag}$ & $\begin{array}{l}\text { 13/keine } \\
77 \% \text { Fusion }\end{array}$ & IV \\
\hline
\end{tabular}

IC) leiden bereits die PEMF-Studien für sich unter der Vielzahl der verschiedenen Stimulationsparameter und unterschiedlichen Behandlungszeiten. Vielen der angegebenen Literaturstellen ermangelt es an der exakten Angabe von Stimulationsparametern, und es ist oft nicht nachvollziehbar, welche Flussdichten, Frequenzen und Amplituden verwendet wurden. In - Tab. 4 sind beispielhaft Stimulationsgeräte aufgelistet, die zurzeit auf dem Markt erhältlich sind, mit den begleitenden technischen Parametern. Aus diesen geht hervor, dass sich diese um mehrere Zehnerpotenzen unterscheiden, so sind $z$. B. bei den Frequenzen Unterschiede um den Faktor $10^{6}$ möglich, die Magnetfelddichten unterscheiden sich um den Faktor von $10^{5} .3$ Arbeiten beschäftigten sich speziell mit dem Einfluss der Feldgrößen auf die Wirksamkeit von elektromagnetischen Verfahren und beschrieben v. a. die niederfrequenten kontinuierlich sinusförmigen Signale im Frequenzbereich von unter $120 \mathrm{~Hz}$ als wirksam mit einem Maximum bei $15-30 \mathrm{~Hz}[55,59,60]$.

Entsprechend den Anwendungsgebieten sind einige Studien mit einem EBMLevel I vorhanden und zahlreiche weitere Studien mit niedrigeren EBM-Leveln. Obwohl die Literatur zahlreich ist

\begin{tabular}{|c|c|c|c|}
\hline Hersteller & Frequenzbereich $[\mathrm{Hz}]$ & Stromstärke $[A]$ & Maximale Magnetfelddichte [mT] \\
\hline 1 & $1-60$ & $0-17$ & $0,001-0,02$ \\
\hline 2 & $1-25$ & & $3-5$ \\
\hline 3 & & & $0,5-5$ \\
\hline 4 & $0,5-300$ & $0,001-1,2$ & 4,5 \\
\hline 5 & $0,1-40$ & $0-3,5$ & $0,001-10$ \\
\hline 6 & $0,5-20$ & & $2-20$ \\
\hline 7 & $0,001-38.000$ & & 15 \\
\hline 8 & $1-10.000$ & $0,00006-0,455$ & Bis 0,15 \\
\hline 9 & $0,2-20$ & $3-5$ & $0,005-0,035$ \\
\hline 10 & $2-20$ & $3-5$ & $0-10$ \\
\hline 11 & $1-1000$ & & $100-180$ \\
\hline \multicolumn{4}{|c|}{$\begin{array}{l}\text { Quelle ist eine Umfrage zu einem Gutachten von Prof. Gabriel zur magnetisch induzierten Elektrostimula } \\
\text { tion, Landessozialgericht NRW, Az L5KR 245/00 }\end{array}$} \\
\hline
\end{tabular}

und sich mit anderen additiven Therapiekonzepten messen kann, wie der Stoßwelle, Ultraschall oder Bone-Matrix-Proteinen, überrascht es dennoch nicht, dass den elektromagnetischen Therapieformen der Durchbruch verwehrt blieb. Dem klinischen Anwender wird durch die Vielzahl der angebotenen Geräte mit ganz unterschiedlichen Parametern die Wahl des optimalen Geräts fast unmöglich gemacht.

\section{Fazit}

Es ist eine „Evidenz" vorhanden, um die elektromagnetischen Verfahren in der Therapie von verzögerten Bruchheilung und Pseudarthrosen einzusetzen oder zur Unterstützung der Knochenheilung bei Arthrodesen des Fußes. Da kaum Nebenwirkungen bekannt sind, lassen sich diese Verfahren bei einem Compliance-fähigen Patienten gut anwenden. Es wird jedoch immer eine Einzelfallentscheidung von Arzt und Patient bleiben, 
welcher Weg am besten einzuschlagen ist. Wünschenswert wäre die Behandlung dieser Patienten in entsprechenden kontrollierten klinischen Studien. Optimal wären placebokontrollierte EBM-Level-I-Studien, jedoch sind auch vergleichende Studien gegenüber anderen Therapieformen, wie Ultraschall, Stoßwelle oder Bone-Matrix-Protein denkbar. Falls sich die elektromagnetische Therapie in entsprechenden Kosten-Nutzen-Analysen (z. B. QUALY-Scores) als effizient behaupten würde, wäre die Entscheidung für den behandelnden Arzt wesentlich leichter, die elektromagnetischen Verfahren als additives Verfahren der Knochenheilung einzusetzen und gegenüber dem Kostenträger zu vertreten.

\section{Korrespondenzadresse}

\section{Dr. S. Hungerer}

BG-Unfallklinik Murnau,

Prof. Küntscher-Straße 8, 82418 Murnau

shungerer@bgu-murnau.de

Interessenkonflikt. Der korrespondierende Autor gibt an, dass kein Interessenkonflikt besteht.

\section{Literatur}

1. Aaron RK, Boyan BD, Ciombor DM et al. (2004) Stimulation of growth factor synthesis by electric and electromagnetic fields. Clin Orthop Relat Res 419: 30-37

2. Akai M, Hayashi K (2002) Effect of electrical stimulation on musculoskeletal systems; a meta-analysis of controlled clinical trials. Bioelectromagnetics 23: 132-143

3. Akai M, Kawashima N, Kimura T et al. (2002) Electrical stimulation as an adjunct to spinal fusion: a meta-analysis of controlled clinical trials. Bioelectromagnetics 23: 496-504

4. Barker AT, Dixon RA, Sharrard WJ et al. (1984) Pulsed magnetic field therapy for tibial non-union. Interim results of a double-blind trial. Lancet 1:994996

5. Bassett CA (1968) Biologic significance of piezoelectricity. Calcif Tissue Res 1: 252-272

6. Bassett CA (1984) [Biomedical and biophysical effects of pulsating electromagnetic fields.] Orthopade 13: 64-77

7. Bassett CA, Becker RO (1962) Generation of electric potentials by bone in response to mechanical stress. Science 137: 1063-1064

8. Bassett CA, Mitchell SN, Gaston SR (1981) Treatment of ununited tibial diaphyseal fractures with pulsing electromagnetic fields. J Bone Joint Surg Am 63: 511-523

9. Bassett CA, Mitchell SN, Schink MM (1982) Treatment of therapeutically resistant non-unions with bone grafts and pulsing electromagnetic fields. J.Bone Joint Surg Am 64: 1214-1220

10. Bassett CA, Valdes MG, Hernandez E (1982) Modification of fracture repair with selected pulsing electromagnetic fields. J Bone Joint Surg Am 64: 888895
11. Borsalino G, Bagnacani M, Bettati E et al. (1988) Electrical stimulation of human femoral intertrochanteric osteotomies. Double-blind study. Clin Orthop Relat Res 237: 256-263

12. Bose B (2001) Outcomes after posterolateral lumbar fusion with instrumentation in patients treated with adjunctive pulsed electromagnetic field stimulation. Adv Ther 18: 12-20

13. Capanna R, Donati D, Masetti C et al. (1994) Effect of electromagnetic fields on patients undergoing massive bone graft following bone tumor resection. A double blind study. Clin Orthop Relat Res 306: 213-221

14. Chang K, Chang WH, Huang S et al. (2005) Pulsed electromagnetic fields stimulation affects osteoclast formation by modulation of osteoprotegerin, RANK ligand and macrophage colony-stimulating factor. J Orthop Res 23: 1308-1314

15. Chang K, Chang WH, Tsai MT et al. (2006) Pulsed electromagnetic fields accelerate apoptotic rate in osteoclasts. Connect Tissue Res 47: 222-228

16. Ciombor DM, Lester G, Aaron RK et al. (2002) Low frequency $E M F$ regulates chondrocyte differentiation and expression of matrix proteins. J Orthop Res 20: 40-50

17. De Haas WG, Watson J, Morrison DM (1980) Noninvasive treatment of ununited fractures of the tibia using electrical stimulation. J Bone Joint Surg Br 62-B: 465-470

18. De Mattei M, Gagliano N, Moscheni C et al. (2005) Changes in polyamines, c-myc and c-fos gene expression in osteoblast-like cells exposed to pulsed electromagnetic fields. Bioelectromagnetics 26 : 207-214

19. Dhawan SK, Conti SF, Towers J et al. (2004) The effect of pulsed electromagnetic fields on hindfoot arthrodesis: a prospective study. J Foot Ankle Surg 43: $93-96$

20. Di Silvestre M, Savini R (1992) Pulsing electromagnetic fields (PEMFs) in spinal fusion: preliminary clinical results. Chir Organi Mov 77: 289-294

21. Dunn AW, Rush GA III (1984) Electrical stimulation in treatment of delayed union and nonunion of fractures and osteotomies. South Med J 77: 1530 1534

22. Eyres KS, Saleh M, Kanis JA (1996) Effect of pulsed electromagnetic fields on bone formation and bone loss during limb lengthening. Bone 18: 505509

23. Fontanesi G, Giancecchi F, Rotini R et al. (1983) Treatment of delayed union and pseudarthrosis by low frequency pulsing electromagnetic stimulation. Study of 35 cases. Ital J Orthop Traumatol 9: 305-318

24. Fontanesi G, Traina GC, Giancecchi F et al. (1986) Slow healing fractures: can they be prevented? (Results of electrical stimulation in fibular osteotomies in rats and in diaphyseal fractures of the tibia in humans). Ital J Orthop Traumatol 12: 371-385

25. Fredericks DC, Nepola JV, Baker JT et al. (2000) Effects of pulsed electromagnetic fields on bone healing in a rabbit tibial osteotomy model. J Orthop Trauma 14: 93-100

26. Fredericks DC, PiehI DJ, Baker JT et al. (2003) Effects of pulsed electromagnetic field stimulation on distraction osteogenesis in the rabbit tibial leg lengthening model. J Pediatr Orthop 23: 478-483

27. Freedman LS (1985) Pulsating electromagnetic fields in the treatment of delayed and non-union of fractures: results from a district general hospital. Injury 16: 315-317

28. Gan JC, Glazer PA (2006) Electrical stimulation therapies for spinal fusions: current concepts. Eur Spine J 15: 1301-1311
29. Glazer PA, Heilmann MR, Lotz JC et al. (1997) Use of electromagnetic fields in a spinal fusion. A rabbit model. Spine 22: 2351-2356

30. Gonzales FL, Arevalo RL, Coretti SM et al. (2005) Pulsed electromagnetic stimulation of regenerate bone in lengthening procedures. Acta Orthop Belg 71: 571-576

31. Gossling HR, Bernstein RA, Abbott J (1992) Treatment of ununited tibial fractures: a comparison of surgery and pulsed electromagnetic fields (PEMF). Orthopedics 15: 711-719

32. Guerkov HH, Lohmann CH, Liu Y et al. (2001) Pulsed electromagnetic fields increase growth factor release by nonunion cells. Clin Orthop Relat Res 384: 265-279

33. Guizzardi S, Di SM, Govoni P et al. (1994) Pulsed electromagnetic field stimulation on posterior spinal fusions: a histological study in rats. J Spinal Disord 7: $36-40$

34. Hartshorne E (1841) On the causes and treatment of pseudarthrosis and especially that form of it sometimes called supernumerary joint. Am J Med 1841: 121-126

35. Heckman JD, Ingram AJ, Loyd RD et al. (1981) Nonunion treatment with pulsed electromagnetic fields. Clin Orthop Relat Res 161: 58-66

36. Hinsenkamp M, Ryaby J, Burny F (1985) Treatment of non-union by pulsing electromagnetic field: European multicenter study of 308 cases. Reconstr Surg Traumatol 19: 147-151

37. Holmes GB Jr (1994) Treatment of delayed unions and nonunions of the proximal fifth metatarsal with pulsed electromagnetic fields. Foot Ankle Int 15: 552-556

38. Ibiwoye MO, Powell KA, Grabiner MD et al. (2004) Bone mass is preserved in a critical-sized osteotomy by low energy pulsed electromagnetic fields as quantitated by in vivo micro-computed tomography. J Orthop Res 22: 1086-1093

39. Inoue N, Ohnishi I, Chen D et al. (2002) Effect of pulsed electromagnetic fields (PEMF) on late-phase osteotomy gap healing in a canine tibial model. J Orthop Res 20: 1106-1114

40. Kennedy WF, Roberts CG, Zuege RC et al. (1993) Use of pulsed electromagnetic fields in treatment of loosened cemented hip prostheses. A doubleblind trial. Clin Orthop Relat Res 286: 198-205

41. Kesani AK, Gandhi A, Lin SS (2006) Electrical bone stimulation devices in foot and ankle surgery: types of devices, scientific basis, and clinical indications for their use. Foot Ankle Int 27: 148-156

42. Kold SE, Hickman J, Meisen F (1987) Preliminary study of quantitative aspects and the effect of pulsed electromagnetic field treatment on the incorporation of equine cancellous bone grafts. Equine Vet J 19: 120-124

43. Kraus W, Lechner F (1972) [Healing of pseudoarthrosis and spontaneous fractures with structure-forming electrodynamic potentials]. Munch Med Wochenschr 114: 1814-1819

44. Lante RW (1850) Cases of ununited fractures treated by electricity. NY State J Med 5: 317-319

45. Lechner F (1973) Proceedings: influencing of impaired fracture healing through electromagnetic fields. Hefte Unfallheilkd 114: 325-327

46. Lechner F, Ascherl R, Uraus W (1981) Treatment of pseudarthroses with electrodynamic potentials of low frequency range. Clin Orthop Relat Res 161: 71-81

47. Li JK, Lin JC, Liu HC et al. (2006) Comparison of ultrasound and electromagnetic field effects on osteoblast growth. Ultrasound Med Biol 32: 769-775 
48. Linovitz RJ, Pathria M, Bernhardt M et al. (2002) Combined magnetic fields accelerate and increase spine fusion: a double-blind, randomized, placebo controlled study. Spine 27: 1383-1389

49. Lohmann CH, Schwartz Z, Liu Y et al. (2000) Pulsed electromagnetic field stimulation of MG63 osteoblast-like cells affects differentiation and local factor production. J Orthop Res 18: 637-646

50. Lynch AF, MacAuley P (1985) Treatment of bone non-union by electromagnetic therapy. Ir J Med Sci 154: 153-155

51. Mammi Gl, Rocchi R, Cadossi R et al. (1993) The electrical stimulation of tibial osteotomies. Double-blind study. Clin Orthop Relat Res 288: 246253

52. Marcer M, Musatti G, Bassett CA (1984) Results of pulsed electromagnetic fields (PEMFs) in ununited fractures after external skeletal fixation. Clin Orthop Relat Res 190: 260-265

53. Marks RA (2000) Spine fusion for discogenic low back pain: outcomes in patients treated with or without pulsed electromagnetic field stimulation. Adv Ther 17: 57-67

54. Massari L, Fini M, Cadossi R et al. (2006) Biophysical stimulation with pulsed electromagnetic fields in osteonecrosis of the femoral head. J Bone Joint Surg Am [Suppl 3] 88: 56-60

55. McLeod KJ, Donahue HJ, Levin PE et al. (1993) Electric fields modulate bone cell function in a density-dependent manner. J Bone Miner Res 8: 977-984

56. Midura RJ, Ibiwoye MO, Powell KA et al. (2005) Pulsed electromagnetic field treatments enhance the healing of fibular osteotomies. J Orthop Res 23: 1035-1046

57. Mooney V (1990) A randomized double-blind prospective study of the efficacy of pulsed electromagnetic fields for interbody lumbar fusions. Spine 15: 708-712

58. O'Connor BT (1985) Treatment of surgically resistant non-unions with pulsed electromagnetic fields. Reconstr Surg Traumatol 19: 123-132

59. Otter MW, McLeod KJ, Rubin CT (1998) Effects of electromagnetic fields in experimental fracture repair. Clin Orthop Relat Res [Suppl] 355: S90-104

60. Rubin CT, Donahue HJ, Rubin JE et al. (1993) Optimization of electric field parameters for the control of bone remodeling: exploitation of an indigenous mechanism for the prevention of osteopenia. J Bone Miner Res [Suppl 2] 8: S573-581

61. Saltzman C, Lightfoot A, Amendola A (2004) PEMF as treatment for delayed healing of foot and ankle arthrodesis. Foot Ankle Int 25: 771-773

62. Savini R, Di SM, Gargiulo G et al. (1990) The use of pulsing electromagnetic fields in posterolateral lumbosacral spinal fusion. J Bioelectricity 9: 9-17

63. Schmit-Neuerburg KP (2001) Indikation und klinische Ergebnisse der magnetfeldinduzierten Wechselstromstimulation verzögert verheilender Frakturen und Pseudarthrosen. Trauma Berufskrankh 3: S66-S72

64. Schnoke M, Midura RJ (2007) Pulsed electromagnetic fields rapidly modulate intracellular signaling events in osteoblastic cells: comparison to parathyroid hormone and insulin. J Orthop Res 25: 933-940

65. Sedel L, Christel P, Duriez J et al. (1982) Results of non unions treatment by pulsed electromagnetic field stimulation. Acta Orthop Scand [Suppl] 196: 81-91

66. Shamos MH, Lavine LS (1967) Piezoelectricity as a fundamental property of biological tissues. Nature 213: $267-269$
67. Shamos MH, Lavine LS, Shamos MI (1963) Piezoelectric effect in bone. Nature 197: 81

68. Sharrard WJ (1990) A double-blind trial of pulsed electromagnetic fields for delayed union of tibial fractures. J Bone Joint Surg Br 72: 347-355

69. Sharrard WJ, Sutcliffe ML, Robson MJ et al. (1982) The treatment of fibrous non-union of fractures by pulsing electromagnetic stimulation. J Bone Joint Surg Br 64: 189-193

70. Simmons JW (1985) Treatment of failed posterior lumbar interbody fusion (PLIF) of the spine with pulsing electromagnetic fields. Clin Orthop Relat Res 193: 127-132

71. Simmons JW Jr, Mooney V, Thacker I (2004) Pseudarthrosis after lumbar spine fusion: nonoperative salvage with pulsed electromagnetic fields. Am J Orthop 33: 27-30

72. Simonis RB, Good C, Cowell TK (1984) The treatment of non-union by pulsed electromagnetic fields combined with a Denham external fixator. Injury 15: 255-260

73. Simonis RB, Parnell EJ, Ray PS et al. (2003) Electrical treatment of tibial non-union: a prospective, randomised, double-blind trial. Injury 34: 357-362

74. Tsai MT, Chang WH, Chang K et al. (2007) Pulsed electromagnetic fields affect osteoblast proliferation and differentiation in bone tissue engineering. Bioelectromagnetics 28: 519-528

75. Yasuda I, Naguahi K, Sata T (1955) Dynamic callus and electricity. J.Bone Joint Surg Am 37A: 12921293

76. Zhang X, Zhang J, Qu X et al. (2007) Effects of different extremely low-frequency electromagnetic fields on osteoblasts. Electromagn Biol Med 26: 167-177 